\title{
Extensión del límite austral de Persea lingue (Lauraceae) al sur de la Provincia de Palena, Región de los Lagos, Chile
}

\section{Extension of the southern limit of Persea lingue (Lauraceae) to the south of the Province of Palena, Region of los Lagos, Chile}

\author{
Cecilia Ezcurra
}

INIBIOMA-CONICET y Universidad Nacional del Comahue, Bariloche, Argentina. cezcurra@crub.uncoma.edu.ar

\begin{abstract}
The finding of Persea lingue (Miers ex Bertero) Nees (Lauraceae) in the south of the province of Palena, Region X of Los Lagos, Chile, is reported. The new locality (Puerto Piedra: $43^{\circ} 26^{\prime} \mathrm{S}, 72^{\circ} 09^{\prime} \mathrm{W}$ ) extends by some $250 \mathrm{~km}$ the southern limit previously described for the species in Chile. This is the first report of $P$. lingue from the North Patagonian rainforest. This species belonging to a mainly tropical genus had previously been associated with the warmer Valdivian, temperate-deciduous and sclerophyllous forests found in Chile further north.
\end{abstract}

Exploraciones botánicas realizadas en el sur de Argentina y Chile con el objetivo de realizar comparaciones entre las floras de los Andes australes y Nueva Zelanda (Wardle et al. 2001, Ezcurra et al. 2008) resultaron en el registro de Persea lingue (Miers ex Bertero) Nees ("lingue", Lauraceae) en los alrededores de la localidad de Puerto Piedra (Chile, X Región de Los Lagos, Prov. Palena, aprox. $43^{\circ} 26^{\prime} \mathrm{S}, 72^{\circ}$ 09' W, 50 m s.n.m.), entre Villa Santa Lucía y la ciudad de Palena. Esta especie arbórea había sido citada para Chile desde la provincia de Quillota hasta la de Chiloé (MuñozSchick1980, Hoffmann 1991, Rodríguez \& Quezada 2001), y desde la V Región de Valparaíso a la X Región de Los Lagos (Grela 2008).

Sin embargo, en los mapas de distribución de Persea lingue publicados en los tratamientos sobre árboles nativos de Chile (Donoso-Zegers 1994, Donoso \& Escobar 2006) y en la Flora de Chile (Rodríguez \& Quezada 2001), el extremo sur del área de la especie figura en los alrededores de Puerto Montt, provincia de Llanquihue, y no incluye Chiloé. Además, los ejemplares de $P$. lingue del herbario de Concepción (CONC, N=173) abarcan solamente desde la latitud $32^{\circ} 47^{\prime} \mathrm{S}$ (Prov. Valparaíso: Quintero) hasta los $41^{\circ} 18^{\prime} \mathrm{S}$ (Prov. Llanquihue: Puerto Varas) y tampoco incluyen la provincia de Chiloé. El material del herbario de Santiago ( $\mathrm{SGO}, \mathrm{N}=83$ ) sigue una distribución similar ya que también abarca mayormente desde $32^{\circ} 53^{\prime} \mathrm{S}$ (Prov. Quillota: Quillota) hasta los $41^{\circ} 55^{\prime}$ (Prov. Llanquihue: Lago Azul). Aunque este material presenta un ejemplar de la provincia de Palena, antiguamente a veces denominada
Chiloé Continental (W Isla Embarcadero o Puerto Nuevo, Río Futaleufú, "Cordillera de Chiloé", s/fecha, leg. Espinosa s/n, SGO 84763), tampoco incluye ejemplares de Chiloé insular. En Argentina la especie se conoce únicamente de un sector de bosque valdiviano situado al norte del Lago Puelo, en el extremo NW de la provincia de Chubut (Pérez Moreau 1984). Allí es una especie rara que crece solamente en un área muy reducida a los $42^{\circ} 05^{\prime} \mathrm{S}, 71^{\circ} 41^{\prime} \mathrm{W}$ y $230 \mathrm{~m}$ s.n.m. (Javier Grosfeld, com. pers.).

Por lo tanto este registro en las cercanías de la ciudad de Palena extiende cerca de $250 \mathrm{~km}$ y más de dos grados al sur el límite anteriormente establecido para la especie en Chile (Rodríguez \& Quezada 2001, Donoso \& Escobar 2006), y marca su límite austral. Esta nueva cita tiene implicancias fitogeográficas, porque Persea lingue es una especie arbórea perteneciente a un género mayormente tropical (Mabberley 1993), y en general es característica de regiones templadocálidas como el bosque esclerófilo de Chile central, el bosque caducifolio templado y el bosque lluvioso valdiviano (Donoso \& Escobar 2006). Esto se evidencia en que presenta el máximo de ejemplares coleccionados entre los $36^{\circ}-39^{\circ} \mathrm{S}$ de su distribución latitudinal (Fig. 1), lo que muestra que es más frecuente y abundante a esas latitudes. El hallazgo de esta especie más al sur, dentro de los límites del bosque lluvioso norpatagónico (sensu Grau 1995), sugiere que a pesar de ser una especie megatérmica, soporta temperaturas más bajas que las que se suponía hasta el momento. Esto también podría tener implicancias en relación a su utilización, porque reflejaría la real amplitud del nicho ecológico de la especie. 


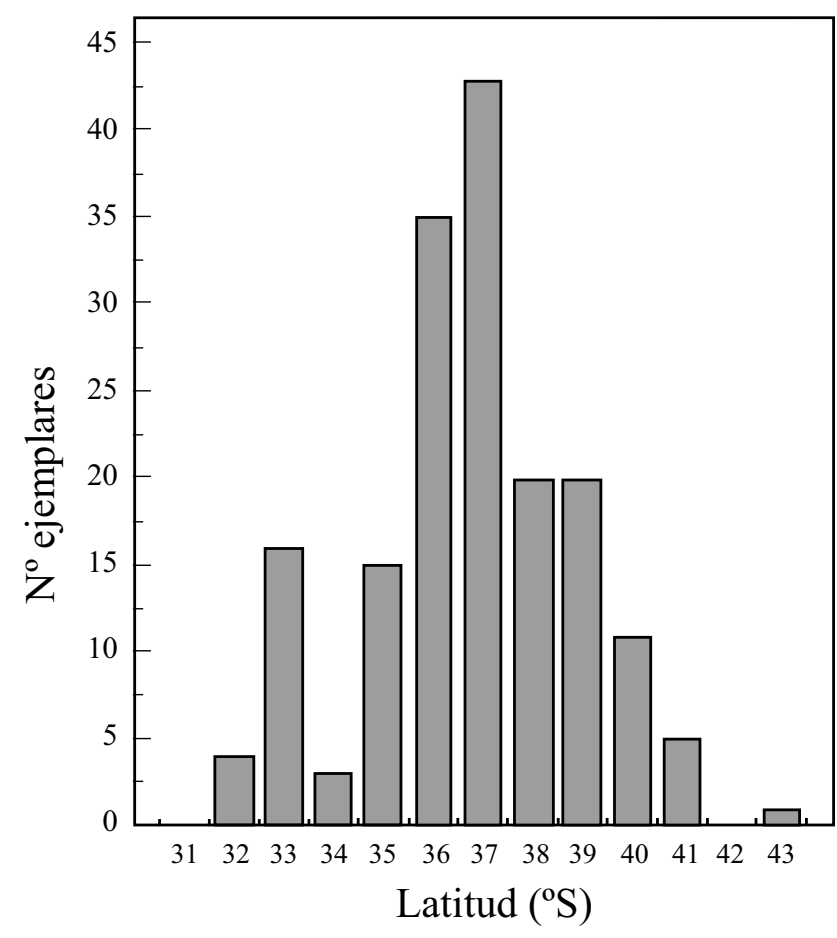

Figura 1. Distribución de Persea lingue en Chile: ejemplares coleccionados en función de la latitud (de base de datos CONC). Los máximos se dan entre los $36^{\circ}-39^{\circ} \mathrm{S}$, lo que refleja que esta especie es característica de formaciones boscosas de regiones templado-cálidas.

Figure 1. Distribution of Persea lingue in Chile: specimens collected in relation to latitude (from CONC data base). Highest numbers between $36^{\circ}-39^{\circ} \mathrm{S}$ show that this species is characteristic of warm-temperate forests.

A pesar de que este registro de Persea lingue en las cercanías de Palena muestra que la especie se extiende más al sur del bosque lluvioso valdiviano, su presencia es rara en esta región. Hasta el momento se han encontrado unos pocos árboles en la localidad de Puerto Piedra, a baja altitud y en la margen del río Futaleufú cerca de su desembocadura en el lago Yelcho, lo que probablemente atempera los fríos más extremos de estas latitudes. Pobladores locales recuerdan que, en la década del 60 , los pocos ejemplares de esta especie existentes en la región se explotaban por su excelente madera para la confección de piezas de embarcaciones (SotoLlanos 2009 com. pers.). El lingue es una especie que fue intensamente cortada en Chile para la producción de tanino y por su madera de gran valor, lo que ha resultado en que actualmente en general sea raro encontrar individuos adultos en los bosques (Donoso \& Escobar 2006).

El ejemplar registrado en Puerto Piedra en 1997 crecía en un remanente de bosque mixto de margen de río con Luma apiculata (DC.) Burret, Drimys winteri J.R. Forst. et G. Forst., Aextoxicon punctatum Ruiz et Pav., Myrceugenia exsucca (DC.) O.Berg y Nothofagus dombeyi (Mirb.) Oerst., y presentaba las características de la especie: forma arbórea elevada (de aprox. $10 \mathrm{~m}$ ) con copa frondosa, tronco grueso y recto (de $35 \mathrm{~cm}$ diámetro) con corteza cenicienta, y hojas simples, alternas, de 6-10 por 3-4 cm, elípticas a ovadas, lustrosas en el haz y corta y densamente pubérulas en el envés, con las venas marcadas, el margen marcadamente revoluto, y el pecíolo canaliculado y pubescente. Exhibía primordios florales en panojas axilares de hasta $4 \mathrm{~cm}$, cubiertos por densa y corta pubescencia pardo-rojiza, y frutos carnosos de 12 por $10 \mathrm{~mm}$, oscuros (Fig. 2).

Además de ese ejemplar encontrado en 1997, en un viaje a Puerto Piedra durante el 2009 se registraron varias otras plantas de menor tamaño aguas abajo, en la costa deshabitada del río más allá de la zona poblada y con construcciones, lo que muestra que el lingue se regenera naturalmente en esta región. Los ejemplares juveniles crecían entremezclados con vegetación natural de olivillo, canelo y patagua, y también al lado de plantas del sauce (Salix fragilis L.) naturalizado en las orillas del río. Estos ejemplares más jóvenes y de sitios menos expuestos al sol en general presentaban hojas mayores, más planas, muy parecidas a las de Persea americana Mill., fragantes al romperlas, con troncos de entre 17 y $3 \mathrm{~cm}$ de diámetro.

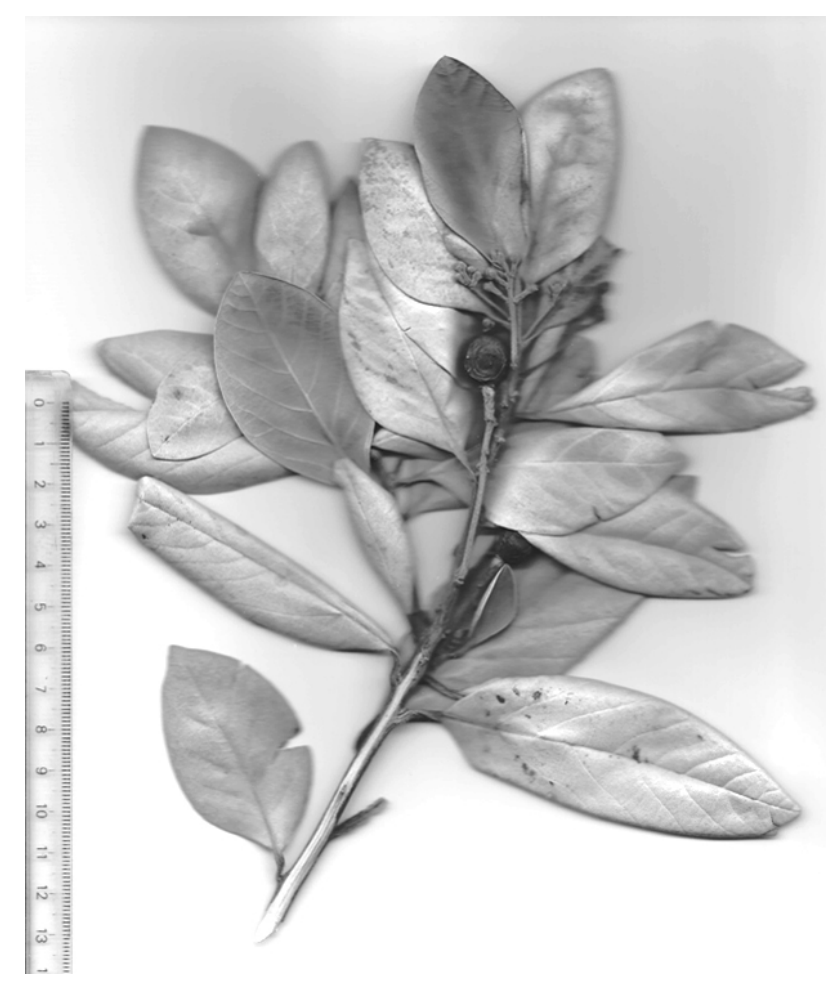

Figura 2. Ejemplar de Persea lingue coleccionado en los alrededores de Puerto Piedra, Prov. Palena, X Región de Los Lagos, Chile.

FIgURE 2. Persea lingue specimen collected in the surroundings of Puerto Piedra, Prov. Palena, Región X of Los Lagos, Chile. 
Material estudiado: CHILE. X Región de Los Lagos. Provincia de Palena. Camino de Futaleufú hacia la Carretera Austral, aprox. a $50 \mathrm{~km}$ de Futaleufú, Puerto Piedra, alrededores del Camping, 30-I-1997, C. Ezcurra 2132, con P. Wardle, S. Wagstaff y M. Caldiz (BCRU); Idem, Puerto Piedra, costa del Camping al lado del río, 10-XI-2009, C. Ezcurra 3602, con D. de Azkue (BCRU); Idem, río abajo aprox. 500 m, 10-XI-2009, C. Ezcurra 3603, con D. de Azkue (BCRU).

\section{AGRADECIMIENTOS}

Agradezco a Clodomiro Marticorena y Mélica Muñoz el envío de información a partir de las bases de datos de los herbarios de Concepción y Santiago, y a Javier Grosfeld los datos detallados sobre la distribución en Argentina. También agradezco a Daniel de Azkue sus comentarios y la ayuda en el campo, a los revisores anónimos sus sugerencias, y a la Agencia Nacional de Promoción Científica y Técnica de Argentina (ANPCyT) el apoyo al PICT 38148 BID 1728/ OC-AR.

\section{BIBLIOGRAFÍA}

Donoso-Zegers, C. 1994. Árboles nativos de Chile, guía de reconocimiento. Marisa Cúneo Ediciones, Valdivia. 216 pp.
Donoso, C. \& Escobar, B. 2006. Persea lingue. En: C. DonosoZegers (ed.), Las especies arbóreas de los bosques templados de Chile y Argentina. Autoecología. Marisa Cúneo Ediciones, Valdivia. 678 pp.

Ezcurra, C., N. Baccalá \& P. WardLE. 2008. Floristic relationships among plant communities of the southern Andes and New Zealand: similarities and biogeographic implications. Annals of Botany 101(9): 1401-1412.

Grau, J. 1995. Aspectos geográficos de la flora de Chile. En: C. Marticorena \& R. Rodríguez (eds.), Flora de Chile 1: 6383. Universidad de Concepción, Concepción, Chile.

Grela, I. 2008. Lauraceae. En: F. O. Zuloaga, M. Belgrano \& O. Morrone (eds.), Catálogo de las Plantas Vasculares del Cono Sur 3: 2939-2404. Missouri Botanical Garden Press, Saint Louis.

Hoffmann, A.E. 1991. Flora silvestre de Chile, zona araucana. Fundación Claudio Gay, Santiago de Chile. 257 pp.

MabBerley, D.J. 1993. The plant book. Cambridge University Press, Cambridge, Inglaterra. $707 \mathrm{pp}$.

Muñoz-Schick, M. 1980. Flora del Parque Nacional Puyehue. Editorial Universitaria, Santiago de Chile. 557 pp.

Pérez Moreau, R. L. 1984. Lauraceae. En: M. N. Correa (ed.), Flora Patagónica 4a: 357-358. Colección Científica INTA, Buenos Aires. Argentina.

Rodríguez, R. \& M. QuezadA. 2001. Lauraceae. En: C. Marticorena \& R. Rodríguez (eds.), Flora de Chile 2(1): 19-25. Universidad de Concepción, Concepción, Chile.

Wardle, P., C. Ezcurra, C. Ramírez \& S. Wagstaff. 2001. Comparison of the flora and vegetation of the southern Andes and New Zealand. New Zealand Journal of Botany 39: 69-108. 